\title{
Improving assessment of urban racial segregation by partitioning a region into racial enclaves
}

\author{
Anna Dmowska ${ }^{1,2}$ and Tomasz F. Stepinski ${ }^{1}$
}

\begin{abstract}
Frequently, a single-value metric is needed to rank urban regions with respect to the level of multiracial segregation or to compare a segregation level of a single urban region at two different times. Assessment of segregation depends not only on a metric used but also on a choice of region's partitioning. The standard practice is to partition the region into singlescale subregions. In the United States, census tracts are the subregions of choice. Census aggregation units including tracts are delineated without a direct regard to racial homogeneity and are in fact heterogeneous. Consequently, using tracts as subdivisions leads to the underestimation of the segregation level of the entire region. Here we propose to partition a region into racial enclaves - units having boundaries that align with transitions between different racial compositions. By reflecting true demographic structure, such units minimize their internal racial inhomogeneity resulting in improved assessment of segregation. Enclaves are defined as aggregates of adjacent census blocks (smallest and the most racially homogeneous census units) of similar composition. In a typical US urban region effective population size of enclaves is an order of magnitude larger than the size of a census tract and yet the segregation calculated based on enclaves is larger than segregation based on census tracts. The proposed methodology is described and applied to a set of 61 largest cities in the U.S. in their metropolitan statistical areas (MSAs) as well as their urban areas (UAs) boundaries using 1990 and 2010 block-level data. The method is compared to the standard methodology using correlations between cities' segregation rankings.
\end{abstract}

\section{Keywords}

Racial geography, residential segregation, racial diversity

\section{Introduction}

Urban populations in the United States are multiracial, and, to a large degree, segregated (Logan et al. 2004; Parisi et al. 2011; Lee et al. 2014). Increasingly, major European cities are also becoming multiracial and segregated (Andersson et al. 2018; Sturgis et al. 2014). Thus, to quantitatively assess a level of racial segregation in American and European urban agglomerations a multiracial approach to segregation is needed. Historically, the topic of racial segregation arose in the United States in the context of segregation of the black minority from the white majority (Massey and Denton 1993; Farley and Frey 1994; Massey and Hajnal 1995). Thus, a biracial approach to quantify racial segregation was the first to be developed (Duncan and Duncan 1955; Lieberson 1969; Massey and Denton 1988; White 1986). Starting from the 1970s, when American society became multiracial, the development of multiracial methods of quantifying segregation had been developed.

A multiracial, segregated urban region can be thought of as spatial mosaic formed by areal patches inhabited by different population groups. This complex pattern cannot be fully described by a single metric of segregation, however, a single metric is what is most frequently needed, for example, to rank urban areas with respect to the level of segregation or to compare a segregation level in a single urban area at two different times. There exist a significant body of literature on single metric quantification of racial segregation in multiracial regions, for a review see (Yao et al. 2019). In particular, Morgan (1975) and Sakoda (1981) proposed a multiracial version of biracial dissimilarity index $D$ (Duncan and Duncan 1955). Later, based on the seminal work by Theil and Finizza (1971) and Theil (1972) Reardon and Firebaugh (2002) proposed measuring segregation of multiracial region using the information-theoretical notion of entropy. Majority of studies on multiracial urban segregation in the United States employed this methodology (Iceland et al. 2002; Fischer 2003; Fischer et al. 2004; Fasenfest et al. 2004; Farrell 2008; Farrell and Lee 2011; Parisi et al. 2011; Lee et al. 2014; Fowler et al. 2016; Ellis et al. 2018; Bellman et al. 2018).

In the entropy-based method, segregation is assessed by comparing the actual spatio-racial distribution of population to a hypothetical reference where all racial subpopulations have the same spatial distribution. The region is divided into constituent census units (most frequently census tracts). Racial diversity is measured as evenness of racial

\footnotetext{
${ }^{1}$ Space Informatics Lab, Department of Geography and GIS, University of Cincinnati, USA

2 Institute of Geoecology and Geoinformation, Adam Mickiewicz University, Poznan, Poland
}

\section{Corresponding author:}

Tomasz F. Stepinski, Space Informatics Lab Department of Geography and GIS, University of Cincinnati, Cincinnati, $\mathrm{OH} 45221$, USA

Email: stepintz@uc.edu 
composition and is expressed by the Shannon (also referred to as Theil's) informational entropy $E$. Racial segregation is measured as a departure from all subpopulations having the same spatial distributions and is expressed by the so-called Information Theory Index $H . H$ is a population-weighted average of differences between the diversity of the entire region and diversities of constituent units normalized by the diversity of the region. In the case of hypothetical reference, all units have the same diversity as the entire region and $H=0 . H>0$ when unit-based diversities diverge from the region's diversity; the larger the average divergence the larger the segregation. $H$ achieves its maximum value of 1 when all units are racially homogeneous.

Both, $D$ and $H$ are aspatial measures, they treat units comprising the region without considering their spatial context and thus suffer from the checkerboard problem (White 1983). Two types of approaches have been proposed to overcome this problem. In one approach (White 1983; Morrill 1991; Wong 1993; Reardon and Sullivan 2004) spatial elements were incorporated into non-spatial indices $D$ and $H$. In another approach (Wong 1999; O'Sullivan and Wong 2007) inherently spatial measures were introduced. However, spatial measures have not been adopted in surveys of urban segregation in the United States (see references earlier in this section), even in the post-2014 surveys despite the availability of open source software for their calculation (Hong et al. 2014).

Another aspect of racial research is the reliance on the data aggregated to areal units of fixed length scale* (blocks, block groups, tracts. etc.). This leads to the Modifiable Areal Unit Problem (MAUP) (Openshaw 1984); all assessments depend on the length scale of units and on locations of their boundaries which are arbitrary with respect to racial composition. The value of $H$ depends on the length scale of units used to subdivide the region (Lee et al. 2008; Iceland and Steinmetz 2003). In general, the more racially homogeneous are the units the larger is the value of $H$ and the higher is the assessment of the region's segregation. Since larger length scale units tend to be less homogeneous, the value of $H$, and thus the assessment of the segregation, decreases with the increase in the length scale of the units (for example, $H$ calculated for a region divided into tracts will be smaller than $H$ calculated for the same region divided into blocks). Thus, using aggregation units it is not possible to calculate unequivocally the value of $H$.

The main goal of this paper is to propose a partition of a region into units having boundaries that align with transitions between different racial compositions. Such units reflect the true demographic structure and do not have a fixed spatial scale. Instead, they have a broad range of scales corresponding to multiple scales of segregation in different parts of the region. This makes possible calculating the unequivocal value of $H$ (or other units-based segregation indexes) that encapsulates regions' segregation better than any value of $H$ based on a division of a region into units having a single length scale.

An idea of partitioning a region into units having boundaries that correspond to transitions between different racial compositions has been previously proposed by Chodrow (2017). His work was motivated by an observation that census-designated places (units larger than tracts) which are sometimes (Fowler et al. 2016) considered as meaningful communities tend to cut through transitions between racial composition and thus are not optimal units for assessing segregation. He proposed to construct more meaningful units by aggregating adjacent census tracts with similar demography regardless to what places they belong to. Our proposed division is similar in spirit, and it follows from a similar observation. We have observed (Stepinski and Dmowska 2019) that census tracts also cuts through transitions between racial composition. Thus, we propose to construct more meaningful units by aggregating census blocks with similar demography regardless of what tracts they belong to. We refer to those aggregated units as enclaves.

Once enclaves are delineated, the segregation of the region could be calculated in a standard manner using the Information Theory Index $H$. However, Roberto (2016) noted that interpreting $H$ as a measure of segregation is misleading because it compares the unit's diversity to the region's diversity rather than comparing the unit's racial composition to the region's composition. Racial composition is a normalized histogram (all bins sums up to 1) of the population with respect to races present in the unit/region and diversity is a measure of evenness of this histogram which does not depend on the order of its bins. Two units may have diametrically different compositions but equal diversities. Instead of $H$, Roberto (2016) proposed using the KullbackLeibler divergence $(K L D)$ (Kullback 1987) as a measure of segregation. $K L D$ measures a difference between two histograms and thus a difference between two compositions. The default measure of segregation in this paper is based on the Jensen-Shannon Divergence or $\operatorname{JS} D(A, B)$ (Lin 1991), where $A$ and $B$ are racial compositions (histograms) of two different enclaves. $J S D$ is a close relative of $K L D$ that has an additional advantage of being symmetric $J S D(A, B)=$ $J S D(B, A)$, whereas $K L D(A, B) \neq K L D(B, A)$. As a control, we also assess levels of segregation using the $H$.

In addition, comparing racial compositions of units with the racial composition of the region does not capture all aspects of segregation because it ignores the topology of racial geography (the checkerboard problem). Although, the introduction of enclaves alleviates the checkerboard problem it does not solve it completely. Given everything else equal, a subpopulation would be perceived as more segregated if it is spatially grouped into a single large enclave instead of several smaller enclaves. We consider topology in our assessment of segregation.

The methodology of delineating racial enclaves and calculating the level of segregation based on the enclaves is given in section 2 . Section 3 presents the results of applying our method to 61 most populous U.S. metropolitan statistical areas (MSAs) and urban areas (UA) in 1990 and 2010. Discussion and conclusions follow in section 4.

\footnotetext{
* The term "scale" or "length scale" in this paper is understood as the spatial extent of a geographic area under study or the so-called measurement scale (Cao and Lam 1997).
} 


\section{Method}

The proposed methodology consists of two steps: (a) delineation of racial enclaves, and (b) calculating segregation metric for the entire population of a given region and segregation metrics for its subpopulations.

\section{Data}

Input data for delineation of racial enclaves are 2010 and 1990 block-level U.S. Census data. We consider five subpopulations: White, Black, Asian (which includes Hawaiian/Pacific Islanders), Hispanics, and others (which include American Indian). All racial subpopulations are nonHispanic. We refer to each sub-population as a race for briefness while remembering that Hispanics label refers to ethnicity.

Blocks are used because they are the smallest and, statistically, the most homogeneous units for which data is available. The most interpretable measure of unit's diversity (racial heterogeneity) is the Hill's number $N_{H}=a^{E}$ (Hill 1973), where $a$ is a base of the logarithm used to calculate the value of unit's entropy $E$. $N_{H}$ can be regarded as an estimate of the effective number of different races present in the unit. Based on the 2010 census data for a set of 61 most populous U.S. metropolitan statistical areas (MSAs) the average value of $N_{H}$ for a block is 1.38 , for comparison, the average value of $N_{H}$ for a tract is 2.90 . By aggregating adjacent blocks of identical or similar composition, we obtain units (enclaves) that are internally stationary (have approximately the same composition on any internal scale from the block scale up) and have boundaries that align well with transitions between different racial compositions. Thus, enclaves are similar in their design to neighborhoods as defined by Spielman and Logan (2013), except that they are mutually exclusive.

\section{Delineation of enclaves}

We achieve delineation of the region into enclaves by aggregating adjacent blocks of the same class where the class is based on racial composition. Several classification schemes have been proposed in the literature (Fasenfest et al. 2004; Johnston et al. 2007; Friedman 2008; Holloway et al. 2012), here we use the classification based on the scheme proposed by Holloway et al. (2012). According to this scheme we classify blocks into three categories: low diversity (L), medium diversity (M), and high diversity (Hdiv). Block is labeled as $\mathrm{L}$ if $E_{\star}<0.483$ and the percentage of the dominant race is $>80 \%$, and it is labeled as Hdiv if $E_{\star}>0.776$, the percentage of the dominant race is $<50 \%$, and the percentage of two most dominant races is $<80 \%$. All remaining blocks are labeled as $\mathrm{M}$. The $E_{\star}=E / \log N$ is the normalized entropy and $N$ is the number of different subpopulation present $(N=5$ in our analysis). Note that the values of thresholds depend on the number of different subpopulations, so the values given here differ from values in Holloway et al. (2012). With five subpopulations there are 11 possible categories of blocks (White, low diversity (WL), Black, low diversity (BL), Hispanics, low diversity (HL), Asian, low diversity (AL), others, low diversity OL, White, medium diversity (WM), Black, medium diversity (BM), Hispanics, medium diversity (HM), Asian, medium diversity (AM), others, medium diversity (OM), high diversity (Hdiv)). Note that low diversity enclaves are approximately monoracial and medium diversity enclaves are approximately biracial.

Classification alone provides visual delineation of a region into enclaves (see Fig. 1). To quantify enclaves numerically we aggregate adjacent blocks of the same class. Such aggregation could be achieved by means of constrained clustering (see, for example, Assunção et al. (2006); Chodrow (2017)), but in order to asses a topological component of segregation we need a data in the form of a grid. Thus, we rasterize block data into a $30 \mathrm{~m}$ resolution categorical grid. Using the grid allows for assessment of the topological component of segregation and also allows for delineation of enclaves using a connected components algorithm (Rosenfeld and Pfaltz 1966) as implemented by Netzel and Stepinski (2013). Note that rasterization of the data does not affect the result of delineation of enclaves; enclaves delineated from blocks or the grid are identical if the basis of delineation is the blocks' classification.

Fig. 1 shows an example of delineation of enclaves in the urbanized area of Los Angeles, CA (UALA-2010) in 2010. The UALA-2010 is inhabited by over 12 million people and is divided into 124,452 blocks. Our procedure results in 19,728 inhabited enclaves. The number of people living in those enclaves varies from 1 to 529,275 . However, majority of people live in large enclaves, 84\% of people live in 906 enclaves with population $\geq 1,000,74 \%$ of people live in 266 enclaves with population of $\geq 4,000$ (where 4,000 is an average population of a census tract), and $50 \%$ of people live in 42 enclaves with population $\geq 50,000$. A populationweighted average population of an enclave in UALA-2010 is 121,574 people. For comparison, the UALA-2010 consists of $\sim 2,400$ tracts. Thus, unlike tracts, enclaves have a wide range of population sizes, but majority of people live in enclaves which are more populous than tracts.

\section{Computing segregation}

Given the multiracial character of U.S. cities and an increasing percentage of non-white populations, we will treat all races equally without identifying a priori a majority race. We view segregation first and foremost as a spatial phenomenon - a physical separation of different races. For segregation to have a meaning for the city as a whole, separated subpopulations need to be sizable. Monoracial city is not segregated, and almost monoracial city, one with a small second race subpopulation isolated in a single location, has only a very small level of segregation. These expectations are met by using the $J S D$ for calculation of segregation, but not by the $H$ (see (Stepinski and Dmowska 2019)). Additionally, we link segregation to a degree of aggregation of people of the same race in a single place. Thus, as mentioned in the introduction, we also consider the aggregation level of the same category enclaves as a factor in calculating a degree of segregation.

In our approach, there are two components of segregation metric, compositional and topological. The compositional component measures a divergence between the racial composition of enclave's population and the composition of the population in the entire region as measured by the $J S D$. The more the enclave's composition diverges from 


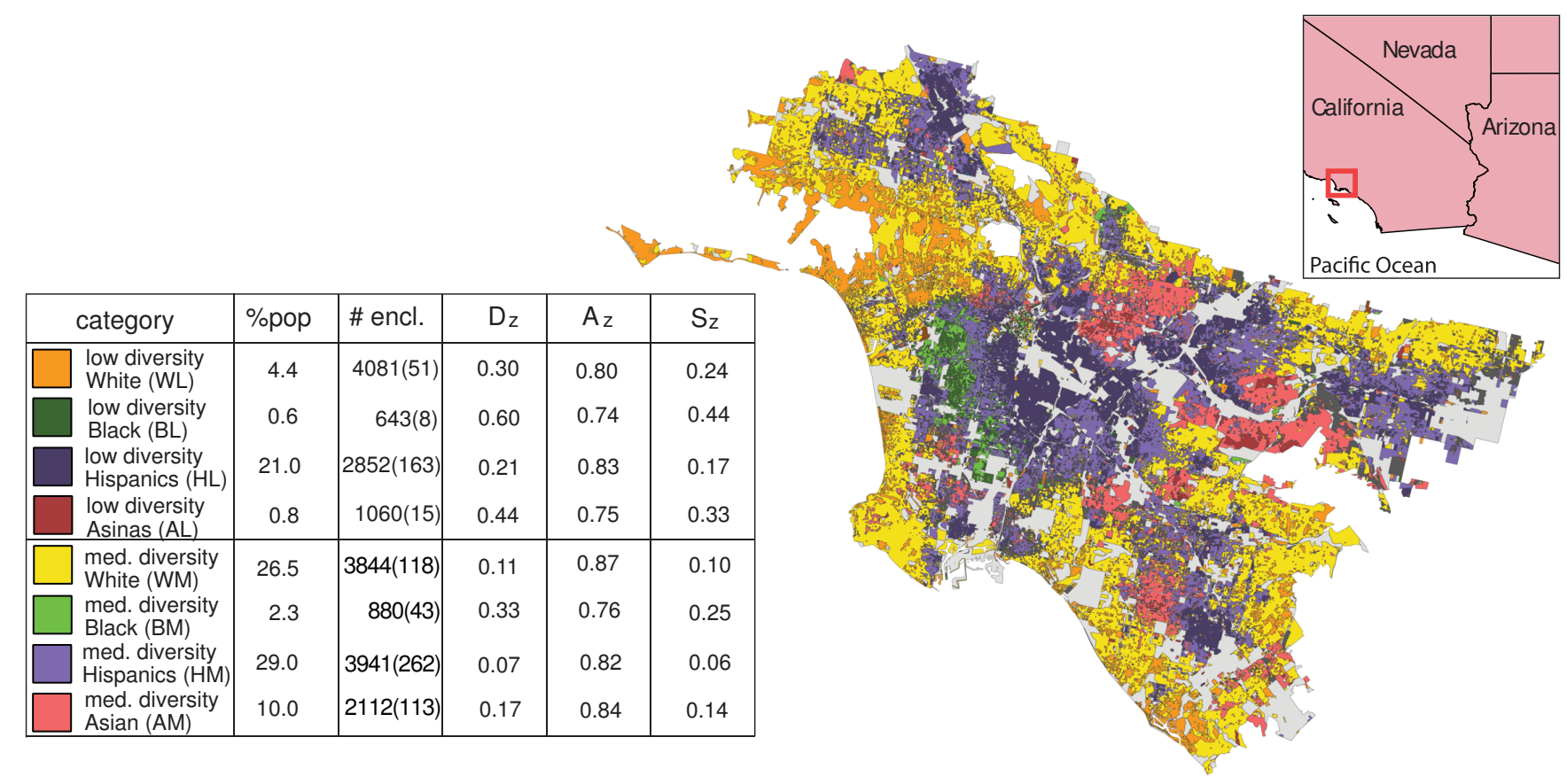

Figure 1. (Left) A map of racial enclaves' categories within the urbanized area of Los Angeles, CA in 2010. Uninhabited patches of the urbanized area are depicted in gray and locations beyond borders of the Los Angeles urbanized area are depicted in white. (Right) A table including the legend to the map and information about categories of enclaves. The third column gives the number of large enclaves: all enclaves (enclaves with population $\geq 1000$ people) of a given category. $D_{z}$ - compositional divergence between a zone and the entire region, $A_{z}$-zone's aggregation index, $S_{z}$-zone's segregation index.

the composition of the region the more segregated it is. Note the relative character of compositional segregation a Black enclave in a predominantly White region is highly segregated, but a Black enclave in the predominantly Black region is minimally segregated. The topological component measures a degree of aggregation. We apply the notion of topological segregation to a zone of enclaves, which can consist of numerous enclaves of the same category. We use a metric called the percentage of like adjacencies $A_{z}$ (McGarigal 2014) to quantify the degree of topological aggregation. $A_{z}=0$ if a zone consists of many small enclaves and $A_{z} \lesssim 1$ if a zone consists of a single, "chunky" enclave. The value of $A_{z}$ depends not only on the number of enclaves but also on shapes of enclaves. It is the calculation of $A_{z}$ that requires gridded data.

The segregation of a zone of enclaves, $S_{z}$, is calculated as a population-weighted average of $J S D$ values between a region and zones' constituent enclaves (denoted by $D_{z}$ ) modulated by zone aggregation (denoted by $A_{z}$ ). The segregation for the entire region, $S_{t}$ is calculated as a population-weighted average of segregation values of its constituent zones. We also calculate segregation metrics, $S_{r}$, for individual races. Details of calculations and specific formulas are collected in Table 1.

A table in Fig.1 shows values of zone divergence $\left(D_{z}\right)$, aggregation $\left(A_{z}\right)$, and overall segregation $\left(S_{z}\right)$ for eight major zones in the Los Angeles urbanized area in 2010. Note that aggregation of all zones is high - as can be inferred from the map in Fig.1, the lion share of the population in each zone is found in just a few large enclaves. The BL zone has the highest value of $D_{z}$ because its composition (mostly blacks) is most dissimilar to the composition $(0.32 \mathrm{~W}, 0.06 \mathrm{~B}$, $0.46 \mathrm{H}, 0.14 \mathrm{~A} .0 .02 \mathrm{O}$ ) of the entire urbanized area. The HM zone has the lowest value of $D_{z}$ because its composition is most similar to the composition of the entire urbanized area. The segregation of the entire urbanized area is a populationweighted average of values in the last column of the table and equals to 0.13 .

\section{Results}

We used the method described in the previous section to calculate segregation metrics for 61 largest US cities. The calculations were performed using 1990 and 2010 census data. For each year, a city spatial extent was taken as either its census-defined MSA boundary or its census-defined urbanized area (UA) boundary. The complete set of results is available in the supplementary file. Table 2 shows a portion of the results pertaining to selected MSAs in 2010. It shows six most segregated MSAs, six least segregated MSAs, and six MSAs in the middle of the segregation ranking. As this is a methodological paper, we don't analyze the results from demographic or social points of view. Instead, we focus on the comparison of the results obtained by the presented methodology with the segregation results obtained by applying the commonly used methodology (tract-based/ $H$-based) to the same data.

Table 2 is divided into several sections. The first two sections give an overall segregation rank and MSA's name. The next two sections give MSA's racial composition and diversity measure (Hill's number). The next section gives values of concentrations for different races in the MSA. For a given race, the concentration is defined as the percentage of this race population living in low diversity enclaves dominated by this race. For example, in Chicago MSA, $60 \%$ of Blacks live in the BL enclaves, since the concentration 
Table 1. Calculating segregation from racial enclaves

\begin{tabular}{|c|c|c|c|}
\hline & enclave & zone & region \\
\hline population & $\begin{array}{l}n_{e, z} \text { is a population in enclave } e \\
\text { belonging to zone } z \\
n_{e, z, r} \text { is a subpopulation } r \text { in } \\
\text { enclave } e \text { belonging to zone } z\end{array}$ & $\begin{array}{l}n_{z} \text { is population in all enclaves of } \\
\text { zone } z \\
n_{z}=\sum_{e} n_{e, z} \\
n_{z, r} \text { is a subpopulation } r \text { in all } \\
\text { enclaves of zone } z \\
n_{z, r}=\sum_{e} n_{e, z, r}\end{array}$ & $\begin{array}{l}n_{t} \text { is the region's entire population, } \\
\text { the sum of populations in all zones } \\
n_{t}=\sum_{z} n_{z} \\
n_{t, r} \text { is the region's entire } \\
\text { subpopulation } r \text {, the sum of } \\
\text { subpopulation } r \text { over all zones } \\
n_{t, r}=\sum_{z} n_{z, r}\end{array}$ \\
\hline divergence & $\begin{array}{l}\text { Divergence between the } \\
\text { composition of an enclave } e \text { and } \\
\text { the composition of the entire region } \\
t \text { is given by the Jensen-Shannon } \\
\text { Divergence } D_{e} \text { : } \\
D_{e}=E\left(\frac{C_{e}+C_{t}}{2}\right)- \\
\frac{1}{2}\left[E\left(C_{e}\right)+E\left(C_{t}\right)\right] \\
\text { where } C_{e} \text { and } C_{t} \text { are compositions } \\
\text { or } e \text { and } t, \text { respectively, and } E\left(C_{x}\right) \\
\text { is an entropy. }\end{array}$ & $\begin{array}{l}\text { Divergence of a zone } z \text { from the } \\
\text { region } t \text { is population-weighted } \\
\text { average of divergences between } \\
\text { compositions of constituent enclaves } \\
\text { and the composition of the entire } \\
\text { region } \\
D_{z}=\sum_{e} \frac{n_{e, z}}{n_{z}} D_{e}\end{array}$ & $\begin{array}{l}\text { Divergence of the entire region } t \text { is } \\
\text { a zone population-weighted average } \\
\text { of zone divergences } \\
D_{t}=\sum_{z} \frac{n_{z}}{n_{t}} D_{z} \\
D_{t} \text { is an approximation of region's } \\
\text { segregation when assessments of } \\
\text { zones' aggregations are not avail- } \\
\text { able. It is a measure of segregation } \\
\text { which does not take into account } \\
\text { spatial component of segregation. }\end{array}$ \\
\hline aggregation & not applicable & $\begin{array}{l}\text { Aggregation } A_{z} \text { of zone } z \text { is given } \\
\text { by the percentage of like adjacencies } \\
\text { (seem the main text) }\end{array}$ & not applicable \\
\hline segregation & $\begin{array}{l}\text { segregation of enclave } e \text { is } \\
S_{e}=D_{e} \\
\text { segregation of a single enclave is } \\
\text { only compositional, it is a measure } \\
\text { of how much the composition of an } \\
\text { enclave differs from the composition } \\
\text { of the entire region. } \\
S_{e} \text { is a number between } 0 \text { (enclave } \\
\text { has the same composition as the } \\
\text { entire region) to } \lesssim 1 \text { (compositions } \\
\text { of enclave and the region are very } \\
\text { different) }\end{array}$ & $\begin{array}{l}\text { Segregation of zone } z \text { is } \\
S_{z}=D_{z} \times A_{z} \text { (9) } \\
\text { Segregation of a zone has a } \\
\text { compositional }\left(D_{z}\right) \text { and spatial }\left(A_{z}\right) \\
\text { components. } \\
\text { Zone segregation is maximum if the } \\
\text { zone consists of only one enclave } \\
\left(A_{z}=1\right) \text { it decreases if the zone } \\
\text { consists of many smaller enclaves } \\
\left(A_{z}<1\right) \text {. }\end{array}$ & $\begin{array}{l}\text { Segregation of subpopulation } \\
\text { in the entire region is an average } \\
\text { of } S_{z} \text { weighted by shares of a } \\
\text { subpopulation in each zone } \\
S_{r}=\sum_{z} \frac{n_{z, r}}{n_{t, r}} S_{z} \\
\text { Segregation of the entire region } \\
\text { is an average of } S_{r} \text { weighted by } \\
\text { shares of region's population in each } \\
\text { subpopulation. It is also an average } \\
\text { of } S_{z} \text { weighted by shares of region's } \\
\text { population in different zones } \\
S_{t}=\sum_{r} \frac{n_{t, r}}{n_{t}} S_{r}=\sum_{z} \frac{n_{z}}{n_{t}} S_{z} \\
(11)\end{array}$ \\
\hline
\end{tabular}

of Blacks for Chicago is $60 \%$. The next to the last section gives segregation metrics for individual races (values of $S_{r}$ obtained from by eq.(10)). The last section of Table 2 gives an overall metric of segregation (values of $S_{t}$ obtained from eq.(11)).

The last row in Table 2 gives average values calculated over the entire set of 61 MSAs. Clearly, in a typical 2010 MSA, Whites were the majority subpopulation. The White subpopulation was also, on average, the most concentrated in monoracial enclaves, while the Black population was the most segregated. Values of correlation between races' shares of population and their concentrations were $0.88(\mathrm{~W})$, $0.80(\mathrm{~B}), 0.93(\mathrm{H})$, and $0.74(\mathrm{~A})$ indicating that as the share of any subpopulation increases so is its tendency to concentrate in monoracial enclaves.

One could expect that race concentration is an indicator of its segregation, however, the values of correlation between race concentration and its segregation are $-0.45(\mathrm{~W})$, $0.85(\mathrm{~B}),-0.03(\mathrm{H})$, and $0.63(\mathrm{~A})$. It is only for Blacks and Asians that larger concentration indicates larger segregation. For Hispanics, there is no correlation between these two metrics, and for Whites higher concentration indicates smaller segregation. The negative correlation for Whites is a consequence of them being the majority race and of a high correlation between concentration and population share. If the share of already dominant subpopulation increases, the divergence between compositions of enclaves and the composition of the entire MSA decreases leading to decreasing of already small segregation of the dominant zone.

The overall segregation metric $S_{t}$ (the last column in Table 2) is a population-weighted average of individual races segregations. Thus, it is a composite metric of MSA segregation. In our sample of 61 MSAs Chicago has the 
Table 2. Racial composition, concentration, and segregation in selected MSAs in $\mathbf{2 0 1 0}$

\begin{tabular}{|c|c|c|c|c|c|c|c|c|c|c|c|c|c|c|c|}
\hline \multirow[b]{2}{*}{ Rank } & \multirow[b]{2}{*}{ MSA name } & \multicolumn{4}{|c|}{ racial composition $\%$} & \multirow{2}{*}{$\begin{array}{l}\text { diversity } \\
N_{H}\end{array}$} & \multicolumn{4}{|c|}{ racial concentration $\%$} & \multicolumn{4}{|c|}{ racial segregation } & \multirow{2}{*}{$\begin{array}{l}\text { total } \\
\text { seg. }\end{array}$} \\
\hline & & White & Black & Hisp. & Asian & & White & Black & Hisp. & Asian & White & Black & Hisp. & Asian & \\
\hline 1 & Chicago, IL & 55 & 16 & 20 & 5 & 3.29 & 53 & 60 & 28 & 2 & 0.11 & 0.33 & 0.18 & 0.11 & 0.161 \\
\hline 2 & Memphis, TN & 46 & 45 & 5 & 2 & 2.73 & 53 & 60 & 4 & 0.5 & 0.14 & 0.16 & 0.14 & 0.10 & 0.148 \\
\hline 3 & New York, NY & 50 & 16 & 23 & 9 & 3.67 & 50 & 27 & 14 & 5 & 0.12 & 0.22 & 0.16 & 0.14 & 0.147 \\
\hline 4 & Birmingham, AL & 65 & 28 & 4 & 1 & 2.43 & 72 & 57 & 8 & 0.9 & 0.10 & 0.25 & 0.17 & 0.08 & 0.145 \\
\hline 5 & Miami, FL & 35 & 19 & 42 & 2 & 3.35 & 31 & 33 & 44 & 0.1 & 0.13 & 0.19 & 0.13 & 0.09 & 0.142 \\
\hline 6 & Houston, TX & 41 & 16 & 36 & 5 & 3.62 & 29 & 20 & 30 & 2 & 0.12 & 0.19 & 0.14 & 0.13 & 0.141 \\
\hline 32 & Austin, TX & 56 & 7 & 32 & 4 & 3.05 & 32 & 1.5 & 16 & 0.3 & 0.07 & 0.1 & 0.10 & 0.06 & 0.079 \\
\hline 33 & Riverside, CA & 38 & 6 & 49 & 5 & 3.29 & 17 & 0.3 & 26 & 0.5 & 0.09 & 0.06 & 0.07 & 0.08 & 0.079 \\
\hline 34 & Buffalo, NY & 81 & 12 & 4 & 2 & 2.12 & 83 & 45 & 1.4 & 0.2 & 0.05 & 0.28 & 0.11 & 0.07 & 0.078 \\
\hline 35 & Kansas City, MO & 75 & 12 & 8 & 2 & 2.40 & 69 & 27 & 5 & 0.4 & 0.05 & 0.22 & 0.13 & 0.07 & 0.077 \\
\hline 36 & Greenville, SC & 73 & 16 & 7 & 1.6 & 2.34 & 66 & 22 & 2.7 & 0.6 & 0.05 & 0.17 & 0.10 & 0.05 & 0.075 \\
\hline 37 & Tucson, AZ & 56 & 3 & 35 & 2.3 & 2.89 & 31 & 0.1 & 25 & 0.4 & 0.06 & 0.05 & 0.10 & 0.05 & 0.075 \\
\hline 56 & Seattle, WA & 72 & 4 & 8 & 11 & 2.91 & 43 & 0.5 & 0.6 & 1.3 & 0.04 & 0.06 & 0.06 & 0.06 & 0.042 \\
\hline 57 & Salt Lake City, UT & 76 & 1 & 17 & 3 & 2.20 & 60 & 0.1 & 2 & 0.1 & 0.03 & 0.05 & 0.07 & 0.05 & 0.040 \\
\hline 58 & Albany, NY & 84 & 7 & 4 & 3 & 1.99 & 80 & 5 & 0.3 & 0.3 & 0.03 & 0.14 & 0.07 & 0.04 & 0.039 \\
\hline 59 & Portland, OR & 77 & 2 & 11 & 6 & 2.35 & 55 & 0.4 & 3 & 0.5 & 0.03 & 0.04 & 0.07 & 0.04 & 0.034 \\
\hline 60 & El Paso, TX & 13 & 2 & 83 & 1 & 1.85 & 0.6 & 0.07 & 74 & 0.001 & 0.07 & 0.06 & 0.03 & 0.05 & 0.033 \\
\hline \multirow[t]{2}{*}{61} & Knoxville, TN & 87 & 6 & 3 & 1 & 1.74 & 85 & 14 & 3 & 0.5 & 0.02 & 0.15 & 0.06 & 0.03 & 0.030 \\
\hline & average MSA & 63 & 13 & 17 & 4 & 2.73 & 53 & 21 & 11 & 0.9 & 0.07 & 0.17 & 0.11 & 0.07 & 0.088 \\
\hline
\end{tabular}

highest value of $S_{t}$. Chicago has also the highest values of $S_{b}$ (Blacks segregation) and $S_{h}$ (Hispanics segregation). Note that Detroit (not shown in Table 2 due to its \#9 rank) has the highest concentration of Blacks (63\% vs. $60 \%$ for Chicago) but a lower value of $S_{b}$ (0.29 vs 0.33 for Chicago) and the lower value of $S_{t}$ ( 0.135 vs. 0.161 for Chicago). The highest concentration of Hispanics is in El Paso (74\%) but their segregation $S_{h}$ is very small (0.03) because they are the dominant race in El Paso. The correlation between $S_{t}$ and diversity metric $N_{H}$ is 0.56 . Thus, in general, more diverse MSAs tend to be more segregated, however, we stress that although low diversity MSAs are always characterized by low segregation, segregation of highly diverse MSAs can vary over the wide range of $S_{t}$ values. Finally, the correlation between values of $S_{t}$ calculated using zone segregations $\left(S_{z}\right)$ that include aggregation component and values of $S_{t}$ without aggregation component is $\lesssim 1$; aggregation does not make much difference because all zones are highly aggregated.

We now compare our methodology with the method based on the partition of a region into census tracts and on using $H$ as a segregation index. The first comparison pertains to the difference in partitions, enclaves versus tracts. For each 2010 MSA in our dataset, we calculated values of $H_{\mathrm{tr}}-$ the Theil's index based on the division of a region into tracts, and $H_{\mathrm{enl}}$ - the Theil's index based on the division of the same region into enclaves. For all MSAs $H_{\text {enl }}>H_{\text {tr }}$, thus, using the enclaves-based partition yields a higher assessment of region's segregation than using the tracts-based partition. This despite the fact that enclaves are, effectively, a largerscale partition that tracts (see the example of UALA-2010).

The relative difference $\delta H=100 \%\left(H_{\mathrm{enl}}-H_{\mathrm{tr}}\right) / H_{\mathrm{tr}}$ is from $7 \%$ to $90 \%$ and the average value of $\delta H$ is $30 \%$. The correlation between values of $\delta H$ and values of $H_{\mathrm{tr}}\left(H_{\mathrm{enl}}\right)$ is $-0.63(-0.38)$ indicating that $\delta H$ tend to decrease with an increasing level of segregation, although the correlation is weak. The value of $\delta H$ depends on a degree to which, in a given MSA, boundaries of tracts cut through transitions between racial compositions. We also calculate the values of $H_{\mathrm{bl}}$ - the Theil's index based on division of a region into blocks; for all MSA $H_{\mathrm{enc}}<H_{\mathrm{bl}}$. This is because although enclaves consist of adjacent blocks with similar composition, the small differences in the composition of its constituent blocks make an enclave more diverse than blocks. However, individual blocks may be too small for robust statistics of racial composition, so it is better to use larger but still homogeneous units like enclaves.

The second comparison pertains to the use of $S_{t}$ (difference in composition) instead of $H=\left(1 / E_{t}\right) \sum_{e}\left(E_{t}-\right.$ $\left.E_{e}\right)\left(n_{e} / n_{t}\right)$ (difference in diversity) as a metric of segregation. The values of the two metrics are not directly comparable as they have different ranges. Although both metrics are defined to have a theoretical range between 0 and 1 , the values of $S_{t}$ are always relatively small because of the way the $J S D$ is defined. The value of $J S D$ is maximal (equal to 1) when two compositions do not contain common subpopulations. This is not possible when measuring divergence between a region and its part (enclave, region, etc.) whose compositions are drawn from the same subpopulations. Thus, we can only compare segregation rankings of MSAs based on $H$ and $S_{t}$. The rank correlation (Spearman's coefficient) between values of $H$ and $S_{t}$ for 612010 MSAs divided into enclaves is 0.64. This means that the order of MSAs sorted by their values of $H$ is noticeable different from the order of MSAs sorted by their values of $S_{t}$. Only two MSAs in the top six shown in Table 2 are in the top six as ordered by $H$.

Given the criticism (Roberto 2016) of $H$ as a measure of segregation (see the introduction section) it follows that segregation ranking based on $S_{t}$ corresponds better to actual differences in the degree of segregation. However, it is not clear whether a shortcoming of $H$ comes from the measuring differences in diversity rather than in composition (as suggested by Roberto (2016) or from the fact that $H$ is normalized by $E_{t}$. We note that the rank correlation between $H \times E_{t}$ and $S_{t}$ is 0.98 . The quantity $H \times E_{t}$ is the not-normalized Theil's index, known in the information theory as the mutual information 
(Stepinski and Dmowska 2019). Thus, it seems that the discrepancy between ranking segregation on the basis $S_{t}$ and $H$ stems not from the fact that the first compares compositions and the second compares diversities, but rather from the fact that $H$ is normalized by the diversity of the entire region. In their seminal paper Theil and Finizza (1971) clearly stated that $H \times E_{t}$ is a measure of segregation and mentioned that such measure can also be used on a relative basis $\left(H \times E_{t}\right) / E_{t}=H$. Somehow, only the relative measure $H$ has been widely accepted and used in the literature despite the existence of several papers (Frankel and Volij 2007; Mora and Ruiz-Castillo 2008; Stepinski and Dmowska 2019) which demonstrated that the not-normalized Theil's index (mutual information) is a more consistent measure.

\section{Discussion}

Attention in research on assessing racial segregation has focused on designing metrics of segregation. The other factor contributing to such assessment - partitioning of region into subregions - received little attention with the exception of realization that values of segregation depend on the scale of subregions. The segregation is underestimated if the subregions are racially heterogeneous. In this paper we focused on delineation optimal subregions called enclaves, that are as racially homogeneous as possible, but, at the same time, large enough to provide good statistics.

In the United States the Census aggregates racial data into same-scale units without explicit attention to racial homogeneity. Thus, all Census units are to some degree racially heterogeneous, with small units like blocks being least heterogeneous and, and bigger units, like tracts, being more heterogeneous. Our proposed solution aggregates blocks of the same or similar racial composition into much larger units - enclaves. Large percentage of enclaves are almost monoracial, some are biracial, and only few are multiracial. Thus, using enclaves minimizes underestimation of segregation. In addition, because enclaves follow natural racial boundaries between different population types, the value of a segregation metric calculated on the basis of enclaves is a better assessment of segregation than any index calculated from single-scale partition.

The results of our computation confirmed that, from the point of view of segregation assessment, partition into enclaves is more optimal than partition into tracts and results in higher values of segregation; the average difference is $30 \%$ but, in regions where tracts are particularly heterogeneous, the difference may be up to $90 \%$. This is important when compiling information on segregation of major metropolitan areas and ranking them by the value of segregation. It is also important when comparing segregation in a single metropolitan areas at different times.

Our method has two ingredients, classification of blocks into diversity/dominant race classes and aggregation of blocks with the same class into enclaves. Using classification raises questions about dependence on arbitrary thresholds, and using aggregation of same-class blocks raises questions about levels of stationarity of enclaves. Both of these concerns are alleviated by demonstrating a posteriori that enclaves are indeed stationary with respect to racial composition. To quantify stationarity of an enclave, $\delta$, we calculated the average value of $J S D$ between all pairs of blocks in an enclave; the smaller the value of $\delta$ the more stationary is an enclave. For low diversity enclaves $\delta=0.03$ 0.05 , for medium diversity enclaves $\delta=0.12-0.14$, and for high diversity enclaves $\delta=0.11$. For comparison, the value of $\delta$ calculated for the entire region is 0.47 . Thus, the classification delineates low diversity (approximately monoracial) enclaves with a high degree of stationarity. Other categories of enclaves are delineated with a fair degree of stationarity.

In addition to the key conclusion described above, our results yield some secondary findings.

(1) Topological component of segregation, which quantifies a level of aggregation of same-class enclaves does not significantly influence the values of segregation index. Calculating values of aggregation index $A_{z}$ for the 2010 data in 61 major MSAs and UAs we observed that values of $A_{z}$ are in all cases high (see, for example, the fifth column in Fig.1 for the case the LAUA-2010) indicating high aggregation of racial zones. This means that the compositional part of the segregation metric is not much modulated by the topological part of the metric. However, a connection between a value of the metric $A_{z}$ and a subjective perception of aggregation in the context of racial segregation is not clear. The metric $A_{z}$ is constructed to measure the entire spectrum of aggregation levels but the lower part of this spectrum is just not present in racial zones. From the urban point of view, we may need to use an aggregation index which is more discriminative between high but different levels of aggregation. This is an issue for future studies.

(2) Rankings of MSAs based on racial segregation depends on which metric is used, the standard $H$ or $S_{t}$. Only two MSAs in the top six shown in Table 2 are in the top six as ordered by $H$. This discrepancy vanishes when a normalized index $H / E_{t}$ is used instead of $H$. This puts additional questions (in addition to those already raised in Frankel and Volij (2007); Mora and Ruiz-Castillo (2008); Stepinski and Dmowska (2019)) about robustness of $H$ for assessment of multiracial segregation.

\section{Acknowledgements}

This work was supported by the University of Cincinnati Space Exploration Institute.

\section{References}

Andersson EK, Malmberg B, Costa R, Sleutjes B, Stonawski MJ and deValk HA (2018) A comparative study of segregation patterns in Belgium, Denmark, the Netherlands and Sweden: Neighbourhood concentration and representation of nonEuropean migrants. European Journal of Population 34(2): 251-275.

Assunção RM, Neves MC, Câmara G and Costa-Freitas C (2006) Efficient regionalization techniques for socioeconomic geographical units using minimum spanning trees. International Journal of Geographical Information Science 20(7): 797-811.

Bellman B, Spielman SE, S R and Franklin (2018) Local Population Change and Variations in Racial Integration in 
the United States, 2000-2010. International regional science review 41(2): 233-255.

Cao C and Lam NS (1997) Understanding the scale and resolution effects in remote sensing and GIS. In: Quattrochi DA and Goodchild MF (eds.) Scale in remote sensing and GIS. Boca Raton, FL: Lewis Publishers, pp. 57-72.

Chodrow PS (2017) Structure and information in spatial segregation. Proceedings of the National Academy of Sciences 114(44): 11591-11596.

Duncan OD and Duncan B (1955) A methodological analysis of segregation indexes. American sociological review 20(2): 210 217.

Ellis M, Wright R, Holloway S and Fiorio L (2018) Remaking white residential segregation: metropolitan diversity and neighborhood change in the United States. Urban Geography 39(4): 519-545.

Farley R and Frey WH (1994) Changes in the segregation of whites from blacks during the 1980s: Small steps toward a more integrated society. American Sociological Review : 23-45.

Farrell CR (2008) Bifurcation, fragmentation or integration? The racial and geographical structure of US metropolitan segregation, 1990-2000. Urban Studies 45: 467-499. DOI: 10.1177/0042098007087332.

Farrell CR and Lee BA (2011) Racial diversity and change in metropolitan neighborhoods. Social Science Research 40(4): 1108-1123.

Fasenfest D, Booza J and Metzger K (2004) Living Together : A New Look at Racial and Ethnic Integration in Metropolitan Neighborhoods, 1990-2000. Technical report.

Fischer CS, Stockmayer G, Stiles J and Hout M (2004) Distinguishing the geographic levels and social dimensions of US metropolitan segregation, 1960-2000. Demography 41(1): $37-59$.

Fischer MJ (2003) The relative importance of income and race in determining residential outcomes in US urban areas, 19702000. Urban Affairs Review 38(5): 669-696.

Fowler CS, Lee BA and Matthews SA (2016) The contributions of places to metropolitan ethnoracial diversity and segregation: Decomposing change across space and time. Demography 53(6): 1955-1977.

Frankel DM and Volij O (2007) Measuring segregation. Technical report, Economics Working Papers (20022016). 180. Iowa State University.

Friedman S (2008) Do declines in residential segregation mean stable neighborhood racial integration in metropolitan America? A research note. Social Science Research 37(3): 920-933.

Hill MO (1973) Diversity and Evenness: A Unifying Notation and Its Consequences. Ecology 54(2): 427-432.

Holloway SR, Wright R and Ellis M (2012) The Racially Fragmented City? Neighborhood Racial Segregation and Diversity Jointly Considered. The Professional Geographer 64: 63-82.

Hong SY, O'Sullivan D and Sadahiro Y (2014) Implementing spatial segregation measures in R. PlosS one 9(1): e113767.

Iceland J and Steinmetz E (2003) The effects of using census block groups instead of census tracts when examining residential housing patterns. Bureau of the Census 5.
Iceland J, Weinberg D and Steinmetz E (2002) Racial and Ethnic Residential Segregation in the United States: 1980-2000. Technical report.

Johnston R, Poulsen M and Forrest J (2007) The geography of ethnic residential segregation: a comparative study of five countries. Annals of the Association of American Geographers 97(4): 713-738.

Kullback S (1987) Letter to the Editor: The KullbackLeibler distance. The American Statistician (41(4): 340-341.

Lee BA, Iceland J and Farrell CR (2014) Is ethnoracial residential integration on the rise? Evidence from metropolitan and micropolitan America since 1980. In: Diversity and disparities: America enters a new century. Russell Sage Foundation, pp. 415-456.

Lee BA, Reardon SF, Firebaugh G, Farrell CF, Matthews SA and O'Sullivan D (2008) Beyond the Census Tract: Patterns and Determinants of Racial Segregation at Multiple Geographic Scales. American Sociological Review 73(5): 766-791.

Lieberson S (1969) Measuring Population Diversity. American Sociological Review 64: 850-862.

Lin J (1991) Divergence measures based on the Shannon entropy. IEEE Transactions on Information Theory 37(1): 145-151. DOI:10.1109/18.61115.

Logan JR, Stults BJ, Farley R and Stults J (2004) Segregation of Minorities in the Metropolis: Two Decades of Change. Demography 41(1): 1-22.

Massey DS and Denton NA (1988) The dimensions of residential segregation. Social forces 67(2): 281-315.

Massey DS and Denton NA (1993) American apartheid: Segregation and the making of the underclass. Harvard University Press.

Massey DS and Hajnal ZL (1995) The changing geographic structure of black-white segregation in the United States. Social Science Quarterly : 527-542.

McGarigal K (2014) FRAGSTATS Help. Documentation for FRAGSTATS 4.0. Technical report.

Mora R and Ruiz-Castillo J (2008) A defence of an entropy based index of multigroup segregation. Technical report, Working Paper 07-76 Economics Series 45 Departamento de Economia, Universidad Carlos III de Madrid.

Morgan BC (1975) The Segregation of Socioeconomic Groups in Urban Areas: A Comparative analysis. Urban Studies 12: 4760.

Morrill RL (1991) On the Measure of Geographical Segregation. Geography Research Forum 11: 25-36.

Netzel P and Stepinski TF (2013) Connected components labeling for giga-cell multi-categorical rasters. Computers and Geosciences 59: 24-30.

Openshaw S (1984) The modifiable areal unit problem, CATMOG (Concepts and Techniques in Modern Geography), volume 38. Norwich: Geobooks.

O'Sullivan D and Wong DW (2007) A Surface-Based Approach to Measuring Spatial Segregation. Geographical Analysis 39(2): 147-168.

Parisi D, Lichter DT and Taquino MC (2011) Multi-scale residential segregation: Black exceptionalism and America's changing color line. Social Forces 89(3): 829-852.

Reardon SF and Firebaugh G (2002) Measures of Multigroup Segregation. Sociological Methodology 32(1): 33-67. 
Reardon SF and Sullivan DO (2004) Measures of spatial segregation. Sociological methodology 34: 121-162.

Roberto E (2016) The Divergence Index: A Decomposable Measure of Segregation and Inequality. arXiv preprint : 1508.01167.

Rosenfeld A and Pfaltz JL (1966) Sequential operations in digital picture processing. J. ACM 13(4): 471-494.

Sakoda JM (1981) A Generalized Index of Dissimilarity. Demography 18: 245-250.

Spielman S and Logan J (2013) Using high-resolution population data to identify neighborhoods and establish their boundaries. Ann. Assoc. Am. Geogr. 103(1): 67-84.

Stepinski TF and Dmowska A (2019) Imperfect melting potAnalysis of changes in diversity and segregation of US urban census tracts in the period of 1990-2010. Computers, Environment and Urban Systems 76: 101-109.

Sturgis P, Brunton-Smith I, Kuha J and Jackson J (2014) Ethnic diversity, segregation and the social cohesion of neighbourhoods in London. Ethnic and Racial Studies 37(8): 1286-1309.

Theil H (1972) Statistical Decomposition Analysis. Amsterdam: North.

Theil H and Finizza AJ (1971) A Note on the Measurement of Racial Integration of Schools by Means of Informational Concepts. The Journal of Mathematical Sociology 1(2): 187193.

White MJ (1983) The measurement of spatial segregation. American journal of sociology 88(5): 1008-1018.

White MJ (1986) Segregation and diversity measures in population distribution. Population index : 198-221.

Wong D (1993) Spatial Indices of Segregation. Urban Studies 30: 559-572. DOI:10.1080/00420989320080551.

Wong DW (1999) Geostatistics as measures of spatial segregation. Urban Geography 20(7): 635-647.

Yao J, Wong DW, J and Minton (2019) Spatial segregation measures: A methodological review. Tijdschrift voor economische en sociale geografie 110(3): 235-250. 